\title{
Columnar defects acting as passive internal field detectors
}

\author{
A. V. Silhanek, ${ }^{1, *}$ L. Civale, ${ }^{1, \dagger}$ and M. A. Avila ${ }^{2, \hbar}$ \\ ${ }^{1}$ Comisión Nacional de Energía Atómica-Centro Atómico Bariloche and Instituto Balseiro, 8400 Bariloche, Argentina \\ ${ }^{2}$ Instituto de Física "Gleb Wataghin," UNICAMP, 13083-970, Campinas - SP, Brazil \\ (Received 24 September 2001; revised manuscript received 6 March 2002; published 3 May 2002)
}

\begin{abstract}
We have studied the angular dependence of the irreversible magnetization of several $\mathrm{YBa}_{2} \mathrm{Cu}_{3} \mathrm{O}_{7}$ and $2 \mathrm{H}-\mathrm{NbSe}_{2}$ single crystals with columnar defects tilted off the $c$ axis. At high magnetic fields, the irreversible magnetization $M_{i}(\Theta)$ exhibits a well-known maximum when the applied field is parallel to the tracks. As the field is decreased below $H \sim 0.02 H_{c 2}$, the peak shifts away from the tracks' direction toward either the $c$ axis or the $a b$ planes. We demonstrate that this shift results from the misalignment between the external and internal field directions due to the competition between anisotropy and geometry effects.
\end{abstract}

DOI: 10.1103/PhysRevB.65.174525

PACS number(s): 74.60.Ge, 74.60.Jg, 74.72.Bk

\section{INTRODUCTION}

It is a well established fact that the presence of columnar defects (CD's) in high-temperature superconductors (HTSC's) enhances the critical current $\left(J_{c}\right)$ due to the strong pinning and the inhibition of thermal wandering when the flux lines lay into these tracks. ${ }^{1,2}$ In the $\mathrm{YBa}_{2} \mathrm{Cu}_{3} \mathrm{O}_{7}$ (Y:123) compound, the directional pinning produced by these correlated structures becomes evident when the angular dependence of $J_{c}$ is studied. ${ }^{3,4}$ In the last decade several works have shown that a sharp peak in $J_{c}(\Theta)$ appears when the applied field $\mathbf{H}$ is aligned with the direction $\left(\Theta_{D}\right)$ of these linear defects ${ }^{1,3,5-8}$ (here $\Theta$ and $\Theta_{D}$ are the angles formed by the crystallographic $c$ axis with $\mathbf{H}$ and the $\mathrm{CD}$, respectively). However, this behavior only holds when $H$ is high enough to ensure that the average vortex direction is parallel to $\mathbf{H}$. At lower fields, both material anisotropy and geometry effects become relevant and modify the vortex orientation, ${ }^{9}$ which consequently may not coincide with that of $\mathbf{H}$. Since maximum pinning occurs when the vortex orientation (given by the internal field $\mathbf{B}$ rather than by $\mathbf{H}$ ) is aligned with the $\mathrm{CD}$, $J_{c}(\Theta)$ should maximize at an angle $\Theta_{\max } \neq \Theta_{D}$. In other words, any misalignment between $\mathbf{B}$ and $\mathbf{H}$ manifests itself as a shift in the angular position of the peak in $J_{c}(\Theta)$ with respect to the tracks direction.

In fact, we have recently shown in Y:123 compounds that, if the anisotropy effect dominates over the geometry effect (as occurs in most cases for HTSC compounds, as we will show below), for fields lower than $10 \mathrm{kOe}$ the peak progressively departs from the track orientation and shifts toward the $c$ axis $^{3}\left(\left|\Theta_{\max }\right|<\left|\Theta_{D}\right|\right)$. In contrast, if the geometry effect dominates over the anisotropy effect (as would be the case in more isotropic materials), the maximum in $J_{c}(\Theta)$ should move toward the $a b$ plane $\left(\left|\Theta_{\max }\right|>\left|\Theta_{D}\right|\right)$. Although the latter effect has not been seen until now, Candia and Civale ${ }^{10}$ have experimentally demonstrated that in the isotropic $\mathrm{Pb}_{0.9} \mathrm{Tl}_{0.1}$ alloy, the sample shape determines the internal field direction. In that study it was shown that, regardless of the direction of $\mathbf{H}$, at low fields the flux lines remain almost locked to the sample normal because the system gains energy by shortening the vortex length.

In this paper we present a study of the angular depen- dence of the irreversible magnetization in several $\mathrm{YBa}_{2} \mathrm{Cu}_{3} \mathrm{O}_{7}$ and $2 \mathrm{H}-\mathrm{NbSe}_{2}$ single crystals with a single set of CD's tilted off the $c$ axis. In particular, we study the misalignment between the external and the internal field directions for several sample aspect ratios and anisotropies. We use the uniaxial pinning of the $\mathrm{CD}$ as a detector of the vortex orientation in the bulk of the samples. We clearly demonstrate the influence of the crystal shape in the determination of the vortex direction and find that the angular behavior of the critical current is well described by the competition between material anisotropy and sample geometry.

\section{THEORETICAL BACKGROUND}

A complete description of the vortex lattice behavior in a superconducting material should include geometry effects, mass anisotropy, vortex-vortex, and vortex-defect interactions. Whenever the system is in thermodynamic equilibrium, the internal field $\mathbf{B}$ is determined by minimization of the free energy $G(\mathbf{B})=F(\mathbf{B})-B^{2} / 8 \pi+(\mathbf{B}-\mathbf{H}) \cdot \mathbf{M} / 2$ [Ref. 9, Eq. (8.60)]. In this expression the magnetization $\mathbf{M}$ and the applied field $\mathbf{H}$ are related by $\mathbf{H}=\mathbf{B}-4 \pi(1-\hat{\nu}) \mathbf{M}$, where $\hat{\nu}$ is the tensor of demagnetization factor. The components of $\hat{\nu}$ at the sample principal axes are $\left(\nu_{x}, \nu_{y}, \nu_{z}\right)$, with $\nu_{x}+\nu_{y}+\nu_{z}=1$. We adopt the notation that $z$ coincides with the crystallographic $c$ axis, and that the $x$ axis is perpendicular to both $c$ and $\mathbf{H}$. Standard minimization of $G(\mathbf{B})$ with respect to $B_{y}$ and $B_{z}$ gives

$$
4 \pi \frac{\partial F}{\partial B_{i}}=B_{i}-\frac{B_{i}-H_{i}}{\left(1-\nu_{i}\right)}, \quad \text { where } \quad i=y, z
$$

In the intermediate-field regime $\left(H_{c 1} \ll H \ll H_{c 2}\right)$ the free energy $F(\mathbf{B})$ is given by [Ref. 9, Eq. (8.54)],

$$
F=\frac{B^{2}}{8 \pi}+\frac{\Phi_{0}}{2\left(4 \pi \lambda_{a b}\right)^{2}} B \epsilon_{\Theta_{B}} \ln \left(\frac{H_{c 2}^{c}}{B \epsilon_{\Theta_{B}}}\right),
$$

where $\Theta_{B}$ is the direction of the internal field, $\epsilon_{\Theta_{B}}$ $=\sqrt{\cos ^{2} \Theta_{B}+\epsilon^{2} \sin ^{2} \Theta_{B}}$, the anisotropy $\epsilon=\sqrt{m_{a b} / m_{c}}<1$, and $\lambda_{a b}$ is the penetration depth for $\mathbf{H} \| c$. 
TABLE I. Irradiation and shape specifications for all the crystals studied.

\begin{tabular}{lcccccccccc}
\hline \hline Crystal & Material & $\epsilon^{-1}$ & $B_{\Phi}(\mathrm{kOe})$ & $\Theta_{D}$ & $t(\mu \mathrm{m})$ & $L_{y}(\mu \mathrm{m})$ & $L_{x}(\mu \mathrm{m})$ & $\nu_{y}\left(\times 10^{-3}\right)$ & $\nu_{x}\left(\times 10^{-3}\right)$ & $f(\hat{\nu}, \epsilon)\left(\times 10^{-3}\right)$ \\
\hline$A$ & $\mathrm{YBa}_{2} \mathrm{Cu}_{3} \mathrm{O}_{7}$ & 7 & 30 & $32^{\circ}$ & 8.5 & 210 & 630 & 40 & 13.5 & +34 \\
$B$ & $\mathrm{YBa}_{2} \mathrm{Cu}_{3} \mathrm{O}_{7}$ & 7 & 30 & $32^{\circ}$ & 20.9 & 715 & 2150 & 29 & 9.7 & +19 \\
$C$ & $\mathrm{YBa}_{2} \mathrm{Cu}_{3} \mathrm{O}_{7}$ & 7 & 57 & $30^{\circ}$ & 11.5 & 1050 & 1050 & 11 & 5.6 & -1.8 \\
$D$ & $\mathrm{YBa}_{2} \mathrm{Cu}_{3} \mathrm{O}_{7}$ & 7 & 22 & $57^{\circ}$ & 4.3 & 381 & 762 & 11.3 & 12 & -67 \\
$E$ & $\mathrm{NbSe}_{2}$ & 3 & 0.5 & $27^{\circ}$ & 7.7 & 765 & 640 & 10.1 & 12 & -63.5 \\
$F$ & $\mathrm{NbSe}_{2}$ & 3 & 0.5 & $27^{\circ}$ & 7.7 & 585 & 640 & 13.2 & 12 & -58 \\
$G$ & $\mathrm{NbSe}_{2}$ & 3 & 0.5 & $27^{\circ}$ & 7.7 & 419 & 640 & 18.4 & 12 \\
\hline \hline
\end{tabular}

We now write all the field dependencies in Eq. (2) in terms of $B_{y}$ and $B_{z}$ and take the derivatives. To that end we replace $B \epsilon_{\Theta_{B}}=\sqrt{\epsilon^{2} B_{y}^{2}+B_{z}^{2}}$. The result is

$$
4 \pi \frac{\partial F}{\partial B_{y}}=B_{y}+\frac{H_{c 2}^{c}}{4 \kappa^{2}} \frac{\epsilon^{2} B_{y}}{B \epsilon_{\Theta_{B}}}\left[\ln \left(\frac{H_{c 2}^{c}}{B \epsilon_{\Theta_{B}}}\right)-1\right],
$$

and an analogous expression for $\partial F / \partial B_{z}$, with $B_{z}$ instead of $B_{y}$ and without the $\epsilon^{2}$ factor in the second term. Here $\kappa$ $=\lambda_{a b} / \xi_{a b}$. By replacing into Eq. (1) we get

$$
\begin{aligned}
& \frac{H_{c 2}^{c}}{4 \kappa^{2}} \frac{\epsilon^{2} B_{y}}{B \epsilon_{\Theta_{B}}}\left[\ln \left(\frac{B \epsilon_{\Theta_{B}}}{H_{c 2}^{c}}\right)+1\right]=\frac{B_{y}-H_{y}}{1-\nu_{y}}, \\
& \frac{H_{c 2}^{c}}{4 \kappa^{2}} \frac{B_{z}}{B \epsilon_{\Theta_{B}}}\left[\ln \left(\frac{B \epsilon_{\Theta_{B}}}{H_{c 2}^{c}}\right)+1\right]=\frac{B_{z}-H_{z}}{1-\nu_{z}} .
\end{aligned}
$$

Multiplying Eqs. (4) and (5) by $\left(1-\nu_{y}\right) B_{z} / B H$ and (1 $\left.-\nu_{z}\right) B_{y} / B H$, respectively, and subtracting, after some algebra we obtain

$$
\sin \left(\Theta_{B}-\Theta\right)=-\frac{f\left(\nu_{y}, \nu_{z}, \epsilon\right) \sin \left(2 \Theta_{B}\right)}{8 \kappa^{2}} \frac{\ln h+1}{h},
$$

where $f\left(\nu_{y}, \nu_{z}, \epsilon\right)=\left(1-\nu_{z}\right)-\left(1-\nu_{y}\right) \epsilon^{2}$, the reduced field $h=H / H_{c 2}\left(\Theta_{B}, T\right)$, the angular-dependent upper critical field $H_{c 2}\left(\Theta_{B}, T\right)=H_{c 2}^{c}(T) / \epsilon_{\Theta_{B}}$, and we replaced $H \approx B$ in the argument of the logarithm.

The result (6) only assumes uniaxial anisotropy and the coincidence of one principal axis with the $c$ axis, and it shows that under those very general conditions the misalignments due to both mass anisotropy and sample geometry have the same field and temperature dependence. The function $f(\hat{\nu}, \epsilon)$, which contains the combined effects of geometry and anisotropy, is the key ingredient of the low-field behavior, as its sign determines whether $\Theta_{B}$ leads or lags behind $\Theta$.

To be more specific, let us consider the typical platelike shape of all the single crystals of both Y:123 and $\mathrm{NbSe}_{2}$ used in this study, with thickness $t$ along the $c$ axis much smaller than the lateral dimensions $L_{x}$ and $L_{y}$. To a first approximation $\nu_{x}=t / L_{x}$ and $\nu_{y}=t / L_{y}$; thus $\nu_{x}, \nu_{y},\left(1-\nu_{z}\right) \ll 1$. If the material is strongly anisotropic and the crystal is not too thin, then $\left(1-\nu_{z}\right)>\left(1-\nu_{y}\right) \epsilon^{2}$; thus $f>0$ and $\Theta_{B}>\Theta$. We will call this the "anisotropy-dominated" situation. In contrast, for thin enough samples of a not too anisotropic material $\left(1-\nu_{z}\right)<\left(1-\nu_{y}\right) \epsilon^{2}$, so $\Theta_{B}<\Theta$. This is what we will call the "geometry-dominated" case. The extreme limit of this case, with an infinite slab $\left(\nu_{x}=\nu_{y}=0\right)$ and ignoring the anisotropy, has been discussed by Klein et al..$^{5}$ It is also worth noting that for an infinite cylinder with axis perpendicular to $\mathbf{H}$, where the geometry effects are expected to cancel out, $\nu_{x}=0$ and $\nu_{y}=\nu_{z}=\frac{1}{2}$, thus $f \propto\left(1-\epsilon^{2}\right)$ and Eq. (6) reduces to the well-known expression for the bulk [Ref. 9, Eq. (8.55)].

Equation (6) allows us to determine which should be the vortex direction $\Theta_{B}$ for a given angle $\Theta$ of the controlled variable $\mathbf{H}$. To check whether this model describes the basic vortex lattice behavior when $\mathbf{H}$ is tilted away from the $c$ axis, we will use the CD's as internal field detectors, taking profit from the fact that $J_{c}$ maximizes when $\Theta_{B}=\Theta_{D}$. Thus, if we know $\Theta_{D}$ and $\Theta_{\max }$ (the angular position of the maximum in $J_{c}$ ), we are able to determine the misalignment $\Theta_{B}-\Theta$ $=\Theta_{D}-\Theta_{\max }$. Although the sign of such misalignment is solely determined by the sign of $f$, its magnitude also depends on additional factors such as $\sin \left(2 \Theta_{B}\right)$ and $\kappa^{2}$. Besides that, the misalignment is strongly temperature and field dependent. It is easy to see from Eq. (6) that $\Theta_{B} \rightarrow \Theta$ for large enough $h$. Throughout this paper we will change each of these factors in order to show that Eq. (6) satisfactorily accounts for the observed properties.

At this point it is important to note that, although the misalignment between $\mathbf{B}$ and $\mathbf{H}$ is a low-field effect, Eq. (6) can only be used in the field range where Eq. (2) is valid, i.e., for $H \gg H_{c 1}$. It turns out that all our data are well described by Eq. (6). However, the very dilute vortex limit is conceptually interesting, and for completeness we will discuss it in the last section.

\section{EXPERIMENTAL DETAILS}

The Y:123 single crystals used in this work were grown by the self-flux method as described in Ref. 11 and exhibit a critical temperature of $T_{c}=92 \mathrm{~K}$. We also performed measurements on single crystals of the layered superconductor $\mathrm{NbSe}_{2}$ with $T_{c}=7.2 \mathrm{~K}$. In all cases, columnar defects off the $c$ axis were introduced by irradiation with $300 \mathrm{MeV} \mathrm{Au} u^{26+}$ ions, using the TANDAR accelerator facility (Buenos Aires, Argentina). Table I summarizes the information about geometrical dimensions, mass anisotropy, dose-equivalent 
matching field $B_{\Phi}$, and angle $\Theta_{D}$ of the CD with respect to the $c$ axis, for all the crystals measured.

The dc magnetization measurements were performed in a commercial superconducting quantum-interference device (SQUID) magnetometer with two pickup coils, and both components (longitudinal $M_{l}$ and transverse $M_{t}$ ) were recorded. Samples could be rotated around the axis perpendicular to both the $c$ axis and the CD. Two measuring procedures were used. The first one consisted of collecting a set of isothermal magnetization loops, each one recorded at a fixed angle $\Theta$. In the second one, which we developed more recently, ${ }^{12}$ the sample is rotated in steps at fixed $T$ and $H$, and $\mathbf{M}$ is recorded at a dense set of orientations (the sample is not rotating during the measuring scan). By appropriate processing, both methods allows us to obtain the irreversible magnetization vector $\mathbf{M}_{i}(H, T, \Theta)$, as described previously. ${ }^{3,10,12,13}$

In general, the relation (given by the critical state Bean model) between $\mathbf{M}_{i}$ and the various components of the anisotropic $J_{c}$ is complicated. However, for thin platelike samples, and as long as $\tan \Theta<L_{y} / t \sim \nu_{y}^{-1}$ (which in all the crystals used in our study is true for almost all field orientations, except in a very narrow angular range near $\Theta=90^{\circ}$ ), due to purely geometrical constraints the following conditions are satisfied: ${ }^{14}$ First, the pinning-related persistent currents flow essentially parallel to the $a b$ planes; thus only one component of $J_{c}$ is involved. Second, $\mathbf{M}_{i}$ is almost normal to the sample surface. Third, the geometrical factor that relates the modulus $M_{i}$ with $J_{c}$ is almost independent of $\Theta$. As a consequence, a measure of $M_{i}(\Theta, H, T)$ is equivalent to a measure of the in-plane $J_{c}(\Theta, H, T)$.

\section{EXPERIMENTAL RESULTS}

\section{A. Anisotropy-dominated case}

Figure 1 shows the angular dependence of the irreversible magnetization for Y:123 crystals $A$ and $B$ at $T=70 \mathrm{~K}$ for several fields. The most evident feature in this figure is the asymmetry of $M_{i}(\Theta)$ around $\Theta=0$ arising in the uniaxial pinning due to the $\mathrm{CD}$. For sample $A$ at $H>10 \mathrm{kOe}$, we observe the well-known peak at the track angular position $\Theta_{D}=32^{\circ}$, and at lower fields the peak progressively shifts away from $\Theta_{D}$ toward the $c$ axis. A more complete set of curves showing the shift for this crystal at various $T$ and $H$ can be found in Ref. 3. A similar behavior is observed in crystal $B$, although the shift turns out to be smaller than that in $A$. These two crystals have the same anisotropy and irradiation conditions, but different shapes. Thus, at the same $T$ and $H$ all factors in Eq. (6) are identical, except for $f(\hat{\nu}, \epsilon)$. As seen in Table I, the difference in demagnetizing factors results in a smaller $f(\hat{\nu}, \epsilon)$ for sample $B$ than for $A$. Hence, the misalignment in sample $B$ is expected to be smaller, as indeed observed.

\section{B. Compensated case}

A striking result predicted by Eq. (6) is that the competing effects (anisotropy and geometry) could be exactly compen-

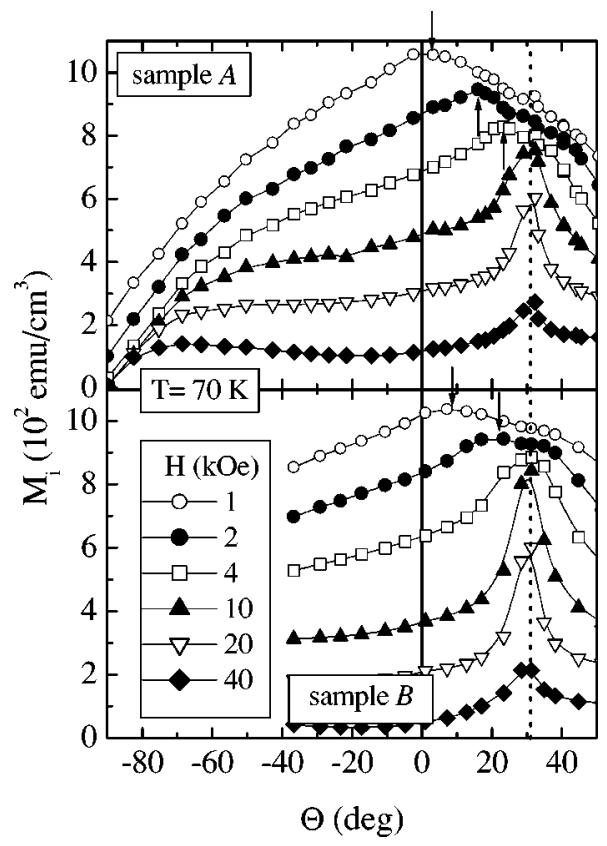

FIG. 1. Angular dependence of the irreversible magnetization as a function of the applied field at $T=70 \mathrm{~K}$ for the samples $A$ and $B$ (Y:123). The arrows indicate the angular position, $\Theta_{\max }$, of the maximum in $M_{i}(\Theta)$ for the lowest fields. For clarity, some curves have been shifted vertically.

sated if one were able to tune the demagnetizing factors and the anisotropy in order to get $f(\hat{\nu}, \boldsymbol{\epsilon})=0$, a condition that is satisfied for $1-\nu_{z}=\left(1-\nu_{y}\right) \epsilon^{2}$. For the $\mathrm{Y}: 123$ single crystals used in this work, with $\epsilon \sim 1 / 7$, this requires extremely thin samples with a big area. Table I shows that crystals $C$ and $D$ almost exactly satisfy this compensating condition, as the absolute values of their $f(\hat{\nu}, \epsilon)$ are, respectively, a factor of $\sim 20$ and $\sim 10$ smaller than in $A$. Figure 2 shows the angular dependence of $M_{i}$ for these two crystals at $T=60 \mathrm{~K}$. For the sake of clarity only a reduced set of fields is shown, and some curves have been shifted vertically. Since the CD orientations $\Theta_{D}$ in samples $C$ and $D$ are different, in order to compare them we set the abscissas as the relative angle $\Theta$ $-\Theta_{D}$. In this figure we clearly observe that the peaks remain locked at the track direction even for the lowest fields, in complete agreement with the expectation. The same behavior was observed for other temperatures.

\section{Geometry-dominated case}

So far, the four samples studied were Y:123 crystals with the same anisotropy but different geometries. On these samples we observed that the peak either shifts in the direction of the $c$ axis or almost does not deviate from the CD direction. As pointed out previously, this behavior arises from the strong anisotropy effect in this material. In order to change the sign of the deviation (i.e., a shift toward the $a b$ plane), we need to reduce the anisotropy effects. (Table I shows that crystal $D$ has $f<0$, thus strictly speaking it is in the geometry-dominated case, but the shift is too small to be detected). 


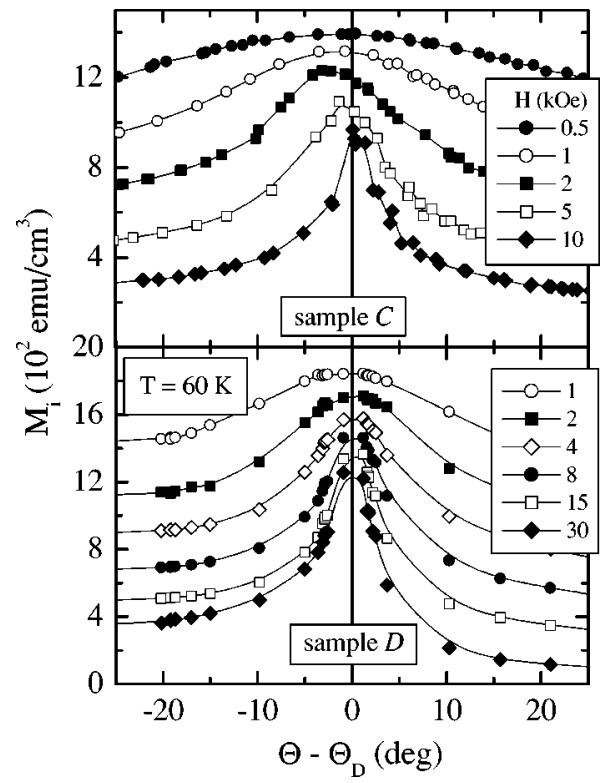

FIG. 2. Irreversible magnetization as a function of the relative angle $\Theta-\Theta_{D}$ for several fields at $T=60 \mathrm{~K}$ in samples $C$ and $D$ (Y:123). For clarity, some curves have been shifted vertically.

To that end we decided to measure $\mathrm{NbSe}_{2}$ single crystals, which have $\epsilon \sim 1 / 3$, making the anisotropy effect about 5 times smaller than that in the Y:123 single crystals. Besides this, very large and thin $\mathrm{NbSe}_{2}$ crystals can be readily found, and they can be easily cut to obtain the desired shape. So we irradiated a rectangular crystal (labeled as sample $E$ ), such that it is in the extreme geometry-dominated case, with $f(\hat{\nu}, \epsilon) \ll 0$. Figure 3(a) shows $M_{i}(\Theta)$ for this sample at $T$

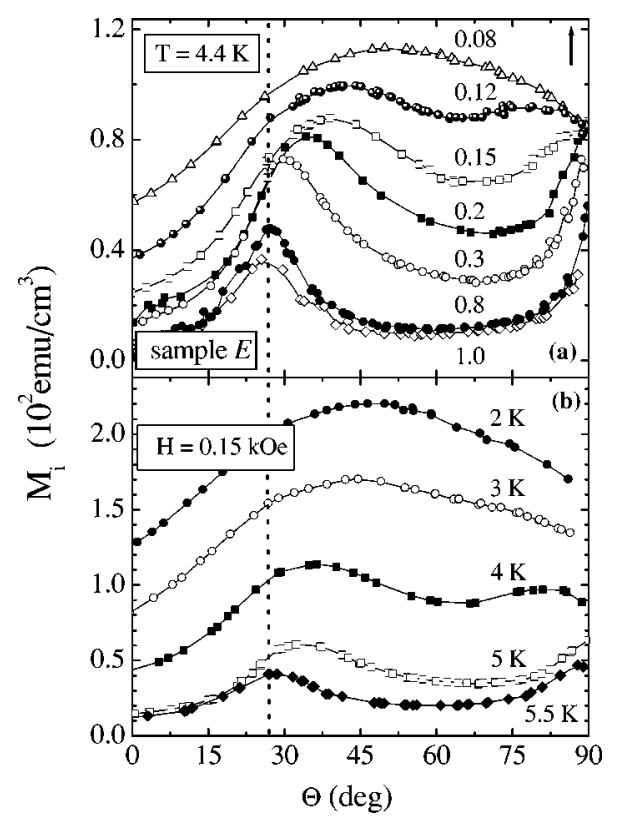

FIG. 3. Angular dependence of the irreversible magnetization for sample $E\left(\mathrm{NbSe}_{2}\right)$ at (a) $T=4.4 \mathrm{~K}$ for several fields (in units of kOe), (b) $H=0.15 \mathrm{kOe}$ for several temperatures. The arrow (up, right) indicates the maximum shift of the peak as predicted by the very-low-field limit (see text).

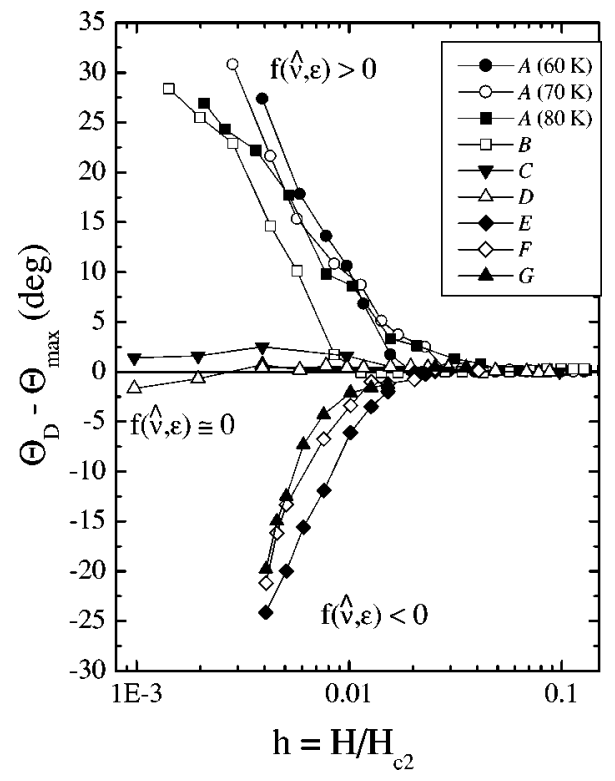

FIG. 4. Deviation in the maximum of the irreversible magnetization with respect to the track direction $\Theta_{D}-\Theta_{\max }$ as a function of $h=H / H_{c 2}$, for all samples studied in the present work.

$=4.4 \mathrm{~K}$ for several $H$. At high fields we observe a peak at the track direction $\Theta_{D}=27^{\circ}$. As the field decreases the peak becomes broader and, in contrast to the Y:123 crystals observed behavior, it progressively moves away from $\Theta_{D}$ toward the $a b$ plane, in agreement with a negative $f(\hat{\nu}, \epsilon)$.

The conclusive evidence that the function $f(\hat{\nu}, \epsilon)$ dominates the behavior of the misalignment $\Theta_{B}-\Theta$ comes from samples $F$ and $G$, which are pieces of crystal $E$. These samples were obtained by cutting the sample $E$ along a line parallel to its shortest side, in such a way that the demagnetizing factor $\nu_{x}$ remains unaltered, but $\nu_{y}$ increases. In this way, the absolute value of the function $f(\hat{\nu}, \epsilon)$ was progressively reduced, i.e., we moved away from the "extreme geometry-dominated case" and approached the "compensated case" (see Table I). According to Eq. (6), the deviation of the maximum in $M_{i}(\Theta)$ for given $H$ and $T$ should become progressively smaller for crystals $F$ and $G$ as compared to crystal $E$. This is in fact observed, as demonstrated in Fig. 4, where $\Theta_{\max }-\Theta_{D}$ for crystals $E, F$, and $G$ is plotted as a function of $h=H / H_{c 2}(T, \Theta)$.

The misalignments for all the Y:123 crystals shown in Figs. 1 and 2 are also included in Fig. 4. Thus, this figure summarizes all the samples studied in the present work at various temperatures and fields. The three possible low-field behaviors are clearly visible: anisotropy-dominated (upward curvature), geometry-dominated (downward curvature) and compensated (almost horizontal curves). It is worth noting that, in all the noncompensated cases and for both materials, the misalignment between $\mathbf{B}$ and $\mathbf{H}$ becomes relevant for fields below a certain characteristic field $H \sim 0.02 H_{c 2}$.

\section{Quantitative test of the model}

The above results clearly demonstrate that the qualitative differences in the low-field behavior are controlled by the 


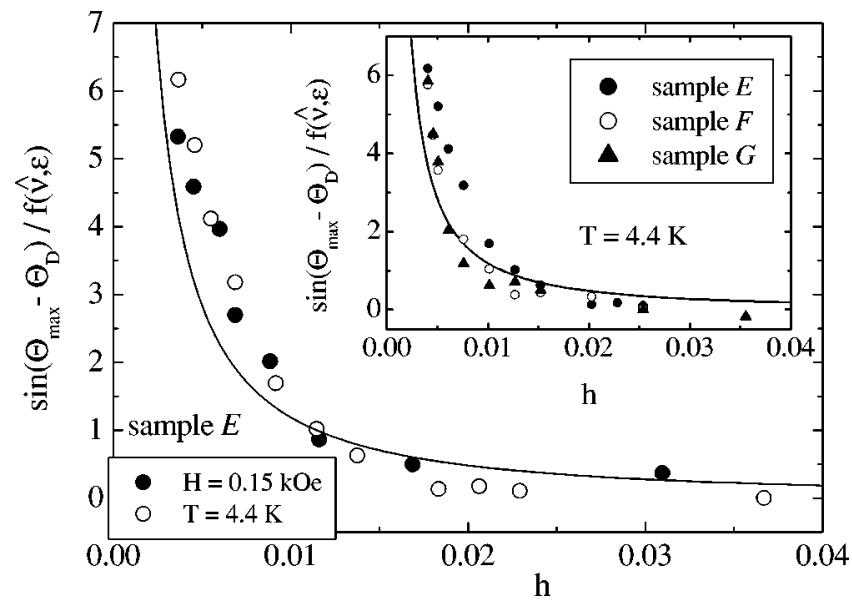

FIG. 5. Main panel: $\sin \left(\Theta_{\max }-\Theta_{D}\right) / f(\hat{\nu}, \epsilon)$ vs $h$ for the two sets of data shown in Figs. 3(a) and 3(b) for the sample E. Solid symbols: $H$ fixed and $T$ swept. Open symbols: $T$ fixed and $H$ swept. Inset: the same scaling shown in the main panel for samples $E, F$, and $G$. The solid line in both, main panel and inset, corresponds to Eq. (6) with $\Theta_{B}=\Theta_{D}=27^{\circ}$ and $\kappa=5.6$.

factor $f(\hat{\nu}, \epsilon)$. We now want to verify whether the $H$ and $T$ dependence of the shift is well described by the model. According to Eq. (6) these two variables appear only through the combination $h=H / H_{c 2}\left(\Theta_{B}, T\right)$. Thus, $\left|\Theta_{D}-\Theta_{\max }\right|$ should increase not only with decreasing $H$ at fixed $T$, as already seen in Figs. 1 and 3(a), but also with decreasing $T$ at fixed $H$, due to the increase in $H_{c 2}(T)$. This second expectation is also verified, as shown in Fig. 3(b) where $M_{i}(\Theta)$ for sample $E$ was plotted at the constant field $H=0.15 \mathrm{kOe}$ for several temperatures.

The equivalence between the variations in $T$ and $H$ is quantitatively verified in the main panel of Fig. 5, where $\sin \left(\Theta_{\max }-\Theta_{D}\right) / f(\hat{\nu}, \epsilon)$ is plotted as a function of $h$ for the two sets of data shown in Figs. 3(a) and 3(b). We observe quite a good scaling, thus confirming that $h$ is the appropriate variable. The upper critical field values $H_{c 2}(\mathrm{kG})=51.8(1$ $-t$ ) were taken from the literature, ${ }^{15}$ thus the superposition of the two curves involves no free parameters. This equivalence between $T$ and $H$ variations is also verified in Y:123 crystals, as demonstrated by the superposition of the three curves of sample $A$ corresponding to different temperatures when plotted as a function of $h$ in Fig. 4.

Finally, we analyze the quantitative effect of the factor $f(\hat{\nu}, \epsilon)$. This factor is a constant for a given sample, so it is the same for all the data in the main panel of Fig. 5. In contrast, in the inset we show the same scaling procedure for the crystals $E, F$, and $G$ at $T=4.4 \mathrm{~K}$, so now $f(\hat{\nu}, \epsilon)$ is different for each sample, while all the other parameters remain identical. We again obtain a good superposition of the data, although the scaling is poorer than in the main panel, probably due to the damage produced in the crystal after each cut process.

The solid line in the main panel of Fig. 5 depicts the expectation of Eq. (6), with $\Theta_{B}=\Theta_{D}=27^{\circ}$ as experimentally determined from the location of the maximum at high fields, and a single fitting parameter $\kappa=5.6$. The same curve is shown in the inset. Although the value $\kappa=5.6$ is smaller than the accepted value ${ }^{15} \kappa \sim 9$, a similar discrepancy was reported by Zhukov et al. when studying the lock-in effect in Y:123 crystals by both twin boundaries ${ }^{7}$ and columnar defects. ${ }^{16}$ Moreover, in previous studies in $\mathrm{Y}: 123$ and $\mathrm{ErBa}_{2} \mathrm{Cu}_{3} \mathrm{O}_{7}$ (Er:123) crystals with tilted CD's we had also found that both the misalignment between $\mathbf{B}$ and $\mathbf{H}$ at low fields $^{3}$ and the width of the lock-in regime ${ }^{12}$ are well described using values of the penetration depth $\lambda$ (or equivalently, of the parameter $\kappa$ in the formalism of the present work) about 3 times smaller than the accepted ones.

\section{THE INFLUENCE OF THE CD's}

It is important to keep in mind that the shift in $\Theta_{\max }$ at low $h$ is not due to the CD's. We are only using them as a passive tool to measure the vortex direction in the bulk of the samples, which is not easy to do by other methods. In fact, the pinning of the $\mathrm{CD}$ is not contained in Eq. (6), which arises from the minimization of a free energy, and thus describes a state of thermodynamic equilibrium. It is obvious, on the other hand, that the uniaxial pinning of these correlated structures is relevant and should be considered. However, the key point in the context of the present study is that the incorporation of the pinning due to the CD into the scenario does not modify the previous results, as we show below.

Correlated pinning is usually included in the analysis ${ }^{6,9}$ by adding to the free energy (2) a term $F_{\text {pin }}$ that accounts for the correction to the vortex self-energy due to the linear or planar defects, and then comparing the energy of alternative configurations. (The procedure is described in Ref. 9, Sec. IX A 1 for the case of twin boundaries and Sec. IX B 5 for the case of CD's.) This additional contribution depends on the orientation of the vortices and the tracks, $F_{\text {pin }}$ $=F_{\text {pin }}\left(\Theta_{B}, \Theta_{D}\right)$, and it is always negative, reflecting the fact that, for $\Theta_{B} \neq \Theta_{D}$, the CD's promote the formation of staircase vortices whose self-energy is lower than that of a straight vortex at the same average orientation. Due to the increase of the core trapped fraction, $F_{\text {pin }}\left(\Theta_{B}\right)$ decreases (becomes more negative) as $\Theta_{B} \rightarrow \Theta_{D}$, and minimizes for that orientation, where the vortex cores are totally trapped into the tracks.

Of course $F_{\text {pin }}\left(\Theta_{B}\right)$ introduces an additional modification to the angle between $\mathbf{B}$ and $\mathbf{H}$. Namely, it will tend to shift the vortices towards $\Theta_{D}$, that is, a kind of effective angular attractive potential towards the CD orientation will develop. But this will not modify Eq. (6). Indeed, if $\mathbf{H}$ is applied at the angle $\Theta=\Theta_{\max }$ such that, in the absence of pinning and according to Eq. (6), the vortices would be at the angle $\Theta_{B}$ $=\Theta_{D}$, the only effect of adding the term $F_{\text {pin }}\left(\Theta_{B}\right)$ (which also minimizes at $\Theta_{B}=\Theta_{D}$ ) will be to deepen the already existing minimum of the free energy at this orientation, without changing the angle.

Let us now suppose that $\mathbf{H}$ is applied at an angle $\Theta$ slightly smaller or slightly larger than $\Theta_{\max }$. In the absence 
of pinning, vortices would respectively orient at angles $\Theta_{B}$ slightly smaller or slightly larger than $\Theta_{D}$, according to Eq. (6). The addition of the term $F_{\text {pin }}\left(\Theta_{B}\right)$ will shift those angles closer to $\Theta_{D}$. In particular, for $\left|\Theta-\Theta_{\max }\right|<\varphi_{L}$, the influence of $F_{\text {pin }}\left(\Theta_{B}\right)$ will be so strong that the system will minimize its free energy by orienting the vortices exactly along the CD. This is the well known lock-in effect, namely, that the internal field remains locked to $\Theta_{D}$ over a finite range of $\Theta$, and $\varphi_{L}(h)$ is called the lock-in angle. In previous works ${ }^{3,12}$ we have extensively studied this effect, which manifests in our measurements as a plateau in $M_{i}(\Theta)$ of width $2 \varphi_{L}$. Note that the center of the plateau coincides with $\Theta_{\max }$. Thus, although the relation $\Theta_{B}$ vs $\Theta$ will be modified by the $C D$, the angle $\Theta_{\max }$, experimentally defined as the maximum in $M_{i}(\Theta)$ or as the center of the plateau where necessary, will still be given by Eq. (6).

A natural consequence of the previous analysis is the fact that the matching field $B_{\Phi}$ does not play any role in our scenario. Having more vortices than CD's or vice versa will modify the strength of the pinning, which is measured by the magnitude of $M_{i}(\Theta)$, but not the angular location of the maximum, $\Theta_{\max }$, which is determined solely by the fact that vortices and CD's are aligned.

As a final comment it is worth mentioning that, although $\varphi_{L} \propto 1 / h$, similarly to the misalignment between $\mathbf{B}$ and $\mathbf{H}$, the physical origin of both effects is totally different. Indeed, in $\mathrm{NbSe}_{2}$ we observe the shift but not the plateau associated with the lock-in, probably because the pinning of the $\mathrm{CD}$ is less effective in this material as compared to the Y:123 and Er:123 compounds.

\section{THE VERY-LOW-FIELD LIMIT}

For completeness, we now consider the very-low-field limit, although it does not apply to our data. In this limit only nearest-neighbor $(\mathrm{NN})$ vortex-vortex interactions need to be taken into account; thus

$$
F \approx \frac{B H_{c 1}\left(\Theta_{B}\right)}{4 \pi}[1+g(\mathbf{B})],
$$

where $H_{c 1}\left(\Theta_{B}\right) \approx H_{c 1}^{c} \epsilon_{\Theta_{B}}$.

In an isotropic material, the sum of the six equal contributions from the $\mathrm{NN}$ gives $g(\mathbf{B})=k r^{-1 / 2} e^{-r}$, where $k$ $=(6 / \ln \kappa) \sqrt{\pi / 2} \sim 1 ; r=a / \lambda$ and $a=\sqrt{\Phi_{0} / B}$ is the vortex lattice parameter. In this limit $r \gg 1$, thus $g(\mathbf{B}) \ll 1$. In the anisotropic case, $g(\mathbf{B})$ must be modified to account for the angular dependence of $\lambda$, and for the distortion of the triangular lattice. Regardless of the details, it is clear that in this case $g(\mathbf{B})$ will still be exponentially small at low enough vortex densities, and the same is true for the derivatives $\left(\partial g / \partial B_{i}\right)$. Thus, replacement of Eq. (7) into Eq. (1) gives

$$
\begin{gathered}
H_{c 1}^{c} \frac{\epsilon^{2} \sin \Theta_{B}}{\epsilon_{\Theta_{B}}}\left[1+\eta_{y}(\mathbf{B})\right]=\frac{H_{y}-\nu_{y} B_{y}}{1-\nu_{y}}, \\
H_{c 1}^{c} \frac{\cos \Theta_{B}}{\epsilon_{\Theta_{B}}}\left[1+\eta_{z}(\mathbf{B})\right]=\frac{H_{z}-\nu_{z} B_{z}}{1-\nu_{z}},
\end{gathered}
$$

where $\eta_{i}(\mathbf{B})$, which account for NN interactions, are exponentially small in the limit of $B \rightarrow 0$.

By taking the ratio of both components, we can calculate the relation between $\Theta$ and $\Theta_{B}$ for the first vortex to penetrate,

$$
\epsilon^{2} \tan \Theta_{B} \approx \frac{\left(H_{y}-\nu_{y} B_{y}\right)\left(1-\nu_{z}\right)}{\left(H_{z}-\nu_{z} B_{z}\right)\left(1-\nu_{y}\right)} \approx \frac{\left(1-\nu_{z}\right)}{\left(1-\nu_{y}\right)} \tan \Theta
$$

So, in the very dilute limit the system is in the geometrydominated case for $\epsilon^{2}\left(1-\nu_{y}\right)>\left(1-\nu_{z}\right)$ and in the anisotropy-dominated case for $\epsilon^{2}\left(1-\nu_{y}\right)<\left(1-\nu_{z}\right)$, exactly the same result that we had found for intermediate fields.

Equation (6) predicts that the misalignment grows indefinitely as $h \rightarrow 0$, even allowing for $\Theta_{B}$ and $\Theta$ to lay in different quadrants. As an extreme case, that equation has no real solution when the absolute value of its right-hand side becomes larger than unity. These clearly unphysical results are just a manifestation of the inapplicability of Eq. (6) at very low fields. As an example, we can estimate the field at which the peak in $M_{i}(\Theta)$ is predicted to coincide with the $a b$ plane $\left(\Theta=90^{\circ}\right)$ for sample $E$. The result is $h \approx 0.0013$, or $H / H_{c 1}$ $\approx 0.09$ [that is, $H \approx 25$ Oe in Fig. 3(a)], clearly below the lower limit of validity. (Note that, due to the strong demagnetizing effects, vortex penetration becomes energetically favorable not at $H_{c 1}$ but rather at a much smaller field $H_{c 1}^{*}$ $\sim\left(1-\nu_{z}\right) H_{c 1} \sim 0.02 H_{c 1}$ for sample E.) In contrast, Eq. (10) indicates that for this sample at very low fields the peak should be observed at $\Theta \approx 88^{\circ}$, as marked with an arrow in Fig. 3(a).

Finally, we must note that the influence of the uniaxial pinning of the $\mathrm{CD}$ turns progressively stronger as $h$ decreases and the term $F_{\text {pin }}$ becomes a significant fraction of the total free energy. At low enough fields the lock-in angle $\varphi_{L} \propto 1 / h$ covers most of the angular range (this is essentially what Klein $e t$ al. ${ }^{5}$ called flux-flop in their early work), thus producing a very broad plateau that turns our method inapplicable to determine $\Theta_{\max }$.

\section{CONCLUSION}

In summary, we performed a detailed study of the influence of anisotropy and sample geometry in the determination of the vortex orientation in bulk superconductors. We showed that these effects are relevant at low fields and become negligible at high fields. On top of that, we developed a model that correctly accounts for the sample shape, mass anisotropy, temperature, and field dependencies of the misalignment between the applied field and the vortex direction. We demonstrated that the sign of the misalignment is solely determined by a function that contains the combined effects of the anisotropy and the demagnetizing factors, and we presented experimental examples of the three possible situations, namely, anisotropy-dominated, geometry-dominated, and compensated cases. We finally discussed the very low- 
vortex-density limit.

An important practical consequence of these results is that studies of the pinning properties of tilted CD's that are based solely on measurements at $\mathbf{H} \| \mathrm{CD}$, or on comparison of this orientation with a few others, give valid information at high fields, but are misleading at low fields: vortices are just not oriented in the right direction. To avoid this problem, a rather complete knowledge of the angular-dependent response is required.

\section{ACKNOWLEDGMENTS}

We are pleased to thank David J. Bishop for providing us with the $\mathrm{NbSe}_{2}$ single crystals and Juan A. Herbsommer for his valuable help in selecting crystals for irradiation. This work was partially supported by ANPCyT, Argentina, PICT 97 No. 01120 and by FAPESP, Brazil, Procs. Nos. 96/ 01052-7 and 96/05800-8. A.V.S. would like to thank the CONICET for financial support.
*Email address: silhanek@cabbat1.cnea.gov.ar

†Present address: Superconductivity Technology Center, Los Alamos National Laboratory, Los Alamos, NM.

${ }^{\ddagger}$ Present address: Ames Laboratory, Iowa State University, Ames, IA.

${ }^{1}$ L. Civale, A. D. Marwick, T. K. Worthington, M. A. Kirk, J. R. Thompson, L. Krusin-Elbaum, Y. Sun, J. R. Clem, and F. Holtzberg, Phys. Rev. Lett. 67, 648 (1991).

${ }^{2}$ M. Konczykowski, F. Rullier-Albenque, E. R. Yacoby, A. Shaulov, Y. Yeshurun, and P. Lejay, Phys. Rev. B 44, 7167 (1991).

${ }^{3}$ A. V. Silhanek, L. Civale, S. Candia, G. Nieva, G. Pasquini, and H. Lanza, Phys. Rev. B 59, 13620 (1999).

${ }^{4}$ A. V. Silhanek and L. Civale, Physica C 341-348, 1227 (2000).

${ }^{5}$ L. Klein, E. R. Yacoby, Y. Wolfus, Y. Yeshurun, L. Burlachkov, B. Y. Shapiro, M. Konczykowski, and F. Holtzberg, Phys. Rev. B 47, 12349 (1993).

${ }^{6}$ V. Hardy, A. Wahl, S. Hèbert, A. Ruyter, J. Provost, D. Groult, and Ch. Simon, Phys. Rev. B 54, 656 (1996).

${ }^{7}$ A. A. Zhukov, G. K. Perkins, J. V. Thomas, A. D. Caplin, H.
Küpfer, and T. Wolf, Phys. Rev. B 56, 3481 (1997).

${ }^{8}$ A. Herbsommer, J. Luzuriaga, L. Civale, G. Pasquini, and G. Nieva, Physica C 304, 112 (1998).

${ }^{9}$ G. Blatter, M. V. Feigel'man, V. B. Geshkenbein, A. I. Larkin, and V. M. Vinokur, Rev. Mod. Phys. 66, 1125 (1994).

${ }^{10}$ S. Candia and L. Civale, Semicond. Sci. Technol. 12, 192 (1999).

${ }^{11}$ F. de la Cruz, D. López, and G. Nieva, Philos. Mag. B 70, 773 (1994).

${ }^{12}$ M. A. Avila, L. Civale, A. V. Silhanek, R. A. Ribeiro, O. F. de Lima, and H. Lanza, Phys. Rev. B 64, 144502 (2001).

${ }^{13}$ A. V. Silhanek, D. Niebieskikwiat, L. Civale, M. A. Avila, O. Billoni, and D. Casa, Phys. Rev. B 60, 13189 (1999).

${ }^{14}$ A. A. Zhukov, G. K. Perkins, Yu. V. Bugoslavsky, and A. D. Caplin, Phys. Rev. B 56, 2809 (1997).

${ }^{15}$ P. de Trey, Suso Gygax, and J.-P. Jan, J. Low Temp. Phys. 11, 421 (1973).

${ }^{16}$ A. A. Zhukov, G. K. Perkins, J. V. Thomas, A. D. Caplin, H. Küpfer, and T. Wolf, Physica C 282-287, 2155 (1997). 